Anfang Juli's wurde an dem Ammersee ein prächtiger Circaëtos gallicus, bei Brannenburg ein ungeheneres $S$ te in adler-Weibchen, (Aquila chrysaëtos $K$. and Bl.,) und auf der Auffhütte zu Moosach bei München ein $11 / 2$ jähriger $\mathrm{Sch}$ reiad ler, ein vermuthlich ächter Falco naevius Linn., erlegt. *)

Die Mauersegler, von welchen die nistenden seit Jahren regelmässig am 26. Juli aus Nürnberg abziehen, waren dort, wie auch zu Ammerndorf und Cadolzburg, noch am 28. anwesend; Tags darauf waren sie jedoch alle verschwunden. Von Norden her durchziehende schwärmten um den hiesigen Kirchthurm noch in den Mittagsstunden des 2. August.

Der sehr verderbliche Nachwinter, ferner der fast unaufhörliche Regen, Gewitter, Hagel-Schauer, Wolkenbrüche und Ueberschwemmungen (also Nässe und Kälte) zur Kielzeit: das Alles liess namentlich ein schlechtes Hühnerjahr erwarten. Gleichwohl hat diese Befürchtung nicht zugetroffen; wenigstens ist mir von vielen Seiten berichtet worden, dass es viele Hühner gebe. Im Aschaffenburgischen wurden schon vor der Erndte sehr starkzählige Hühnerketten angetroffen. (Finer meiner Freunde zählte daselbst auf seinem Jagdbogen am 18. Juli deren schon 8, ohne noch ein einziges Paar gelter Hühner gefunden zu haben.) Auch im Ansbachischen und in hiesiger Gegend liefert die Niederjagd guten Ertrag an Hühnern; und werden gelte Paare wenigstens nicht häufiger als sonst angetroffen. Doch sind noch zu Anfang Septembers die Jungen sehr "gering“, (klein:) was eine sehr nalürliche Folge des Nachwinters ist, welcher die schon zuvor gepaarten Hühner nöthigte, wieder in Ketten zusammenzufallen, und so die Bruten bedeutend verspätete.

Schliesslich noch die Bemerkung: dass am 27. August, bei einem starken Gewilter ein Wendehals zu Cadolzburg Nachts $10 \mathrm{Uhr}$ an die erleuchteten Fenster eines Hauses, flog und gefangen wurde; und dass ich von dort am 5. September einen, soeben ausgeflogenen, jungen Gimpel erhielt.

\title{
Vermischtes über Vögel in der Umgegend von Mainz.
}

Von

Notar Dr. Bruch.

1. Cursorius isabellinus. Dem in Heft I d. "Journ." als "zweites d e $\mathrm{tsches}$ " Exemplar angeführten $C$. isabell. aus Mecklenburg muss ich das Recht auf diese Rangordnung bestreiten; und zwar zu Gunsten eines fruher vorgeliommenen, welches schon über 12 Jahre lang in

*) Im Winter $1851 / 52$ erhielt das Kabinet in München 3 prächtige $S$ te ina dler aus der Kamsau bei Berchtesgaden, welche, nachdem sie mit noch einigen Genossen dieser Art beträchtlichen Schaden an den Gemsen verursacht hatten, in Fuchs-Eisen gefangen wurden. Einer hatte sich durch seine Fluchtanstrengungen die ganze Sehnenlage des Tarsus abgeschärft, so dass der entblösste Knochen zu Tage lag. 
der Sammlung zu Wiesbaden steht. Dasselbe ist bei EItfeld erlegt werden, jedoch wahrscheinlich auf der linken Rheinseite: weil sich der, von dem Vogel sehr geliebte Triebsand nur da in Menge vorfindet. Ich, wie Andere, trafen denselben dort mehrfach: indem er die, bekanntlich im Laufen und Fliegen so äusserst raschen Sandlaufläfer (Cicindela) unter lautem Schreien verfolgte. Ich sah übrigens jedoch nur junge. ob das aber "geborene Deutsche" gewesen sein mögen? -

2. Da, wo jener Sand reichlicher mit Wasser gesättigt erscheint, (wie in dem benachbarten Haidesheim), da ist eine sehr ergiebige Becassinen-Jagd, welche mir gelegentlich manchen seltneren Vogel zugeführt hat: zum Theil auch solche aus ganz anderen Ordnungen. So erhielt ich z. B. im letzten April von da einen männlichen alte n Falco rufpes, der sonst hier noch nicht vorgekommen war.

3. In derselben Gegend, auf dem Rheine, wurde im Jahre 1847 ein prachtvoller alter schwarzer Schwan, Cygnus atratus, geschossen, der in unserer Sammlung zu sehen ist. *) Es verlautete zwar, dass um dieselbe Zeit ein solcher Schwan dem Herrn v. Rothschild durchgegangen sei; aber zugleich auch, dass noch 2 andere Vögel dieser Art auf dem Rheine erlegt worden seien. $O b$ und wie etwa diese mehreren Individuen in Beziehungen zu einander gestanden haben können, dürfte sich'wohl schwer ermitteln lassen.

4. Junge Exemplare von Cygnus olor kommen alljährlich im Freien hier vor, wo sie alsdann sich wie ächt-wilde betragen: obgleich sie vermuthlich auch nur Flüchtlinge aus irgend einem Parke sind, wie Anas sponsa, wie Anser aegyptiacus $\mathbf{u}$. dergl. Selbst in meiner Gegenwart wurde auf dem Rheine ein B astard von Anas moschata geschossen; und wir besitzen in hiesiger Sammlung einen Bastard von Anser canadensis und $A$. cygnoides. I ch bin daher bei dieser Betrachtung zugleich an Lord Cochrane's einzelnen schwarzen Schwan in Chile erinnert worden. **t)

Die Frage über dieses D omesticitäts-Verhältniss lässt sich aber nicht überall so leicht abmachen. So z. B.:

5. Auf mehreren Rhein-Inseln findet man den gewöhnlichen Fasan, Phasianus colchicus; und zu Oppenheim hat ein Gutsbesitzer eine grosse Zucht davon. Die Vögel verbreiten sich von dort in die Nachbarschaft, und erhalten sich dann oft Jahre lang olne besonderen Schutz. So lange hierbei nur von $P h$. colchicus die Rede ist, werden solche Exemplare für wilde angesehen. Krommt jedoch ein

*) In der "Sammlung" der Mainzer naturforschenden Gesellschaft, welche namentlich durch Hrn. Bruch's Bemühungen so reichhaltig an Vögeln aus der, von ihm seit langer Zeit vorzugsweise beachteten Familie der Möven geworden ist, und welche er hierin fortwährend durch eigene nene Erwerbungen zu vermehren sucht. Er hat daher auch schon jetzt bedeutende Ergänzungen zu seiner, in Heft II d. "Journ." gelieferten monographischen Uebersicht derselben in Aussicht gestellt.

D. Herausg.

**) Derselbe war nämlich dem genannten Eigenthümer yon seinem Landsitze in der Nähe von Valparaiso (oder Valdivia?) enthohen. Da lebte er dann eine längere Reihe von Jahren hindurch auf den grossen benachbarten Gewässern, unter den zahlreich dort einheimischen, kleineren weissen Schwänen mit schwarzem Kopfe und Halse, Cygnus nigricollis. 
Gold- oder Silber-Fasan (Ph. pictus oder Ph. nychthemerus) darunter ror: dann ist dieser ein Flüchtling.

6. Das rothe Repphuhn, Perdix rufa, ist schon zweimal in grösseren Gesellschaften bei uns getroffen worden, uber deren Herkunft Niemand Bescheid wusste. Eine dergleichen, von welcher ein Stïck erlegt wurde, sämmtlich Weibchen, bestand nach meiner Ansicht nicht aus gewöhnlichen Flüchtlingen, sondern war auf der "MännerJagd $^{\text {" }}$ begriffen: wie ich diess auch bei anderen Vögeln öfters beobachtet habe. Auf dem Zuge müssen manche einen Führer haben.

7. An denjenigen wasserreicheren Stellen, wo ehemals der eigentliche Rheinstrom floss, und welche daher noch dermalen der Alt$r$ hein ${ }^{*}$ genannt werden, finden sich oft ganze $\mathrm{Z}$ ìg e von hier sonst nicht einheimischen Vögeln ein: wie Ibis falcinellus, Phoenicopterus antiquorum. (Der Ort, wo bereits im April des Jahres 1728 der crste. Flamingo geschossen wurde, kann übrigens nicht "Alzei ${ }^{*}$ gewesen sein, welches yom Rheine ziemlich fern liegt, auch keinen Altrhein in seiner Nähe hat. Es muss wohl heissen: Alsheim !)

8. Manche dergleichen Fremdlinge haben aber sogar da genistet; so z. B. Cygnus musicus.

9. Andere, die verwundet oder sonst krank waren, blieben desshalb zurick: wie Mergus merganser und die nordischen Enten. Wieder andere lassen sich in der That heimisch da nieder. *) So die CircusArten und die Milane, von welchen Milvus ater von Jahr zu Jahr häufiger und so dreist, wie am Nil, wird. Einige Dampfboot-Conducteure pflegen ihn daher an gewissen Stationen durch einen Pfiff herbeizulocken, um ihn dann einen Frass hinzuwerfen, den er sogleich erhascht.

10. Noch will ich bei dieser Gelegenheit erwähnen, dass günstige Zufalligkeiten, (oder vielleicht die Kriegsunruhen in Ungarn?) uns eines Tages eine ganze Familie der Muscicapa parva zugefuihrt haben.

Mainz, den 13. Juni 1853.

Die Verbreitums der vägel bel ung frifher und jetzt. - Man hat seil einiger Zeit mit Recht mehr und gründlicher, als fruher, darauf geachtet: dass manche südlicbere Vogelarten, freilich meistens nur solche aus der Zahl der kleineren, jetzt offenbar zahlreicher an Individuen werden, sich also mehr nach dem Norden verbreiten. So unter anderen, wie bekannt, besonders Ruticilla thitys auf recht sichtliche Weise.

Hiermit im Zusammenhange steht natürlich aber die Frage: welche andere sich, umgekehrt, jetzt mehr aus Deutschland etc. gegen

*) Das mag wohl auch Mergus merganser gethan haben: wenn es nicht seit bereits längerer Zeit geschehen, oder vielleicht gar von jeher der Fall gewesen und früher nur übersehen worden ist. Denn er brütet ja regelmässig, wiewohl nicht gerade häufig, auf mehreren grossen Wassern anderer, noch südlicher gelegener Theile Deutschlands: auf dem Bodenseee, manchen grossen Seeen und Teichen Bayerns etc.; namentlich auf derien in Gebirgen, wo auch die rauhere Temperatur und der kürzere Sommer mehr denen seiner gewöhnlichen, höher nordischen Heimath ähnlich sind.

Gl pger. 